\title{
Validação preliminar de uma versão do Caregiver Reaction Assessment em um contexto de pacientes oncológicos em internação
}

\author{
Preliminary validation study of a Brazilian version of the Caregiver \\ Reaction Assessment in a context of oncologic inpatients \\ Suzane Pereira Busatta', Renata Alexandre Ferreira', Luiz Gustavo Klumb-Kiesow², Hudson W. de Carvalho²
}

\section{RESUMO}

Objetivo: Adaptar para o Brasil uma versão portuguesa do Caregiver Reaction Assessment (CRA) e gerar indicadores preliminares de validade e fidedignidade para sua aplicação em cuidadores de pacientes oncológicos internados. Métodos: Participaram voluntariamente 53 cuidadores, que responderam a um questionário sociodemográfico, ao CRA e à Escala de Bem-Estar Psicológico (EBEP). A unidimensionalidade e a homogeneidade dos escores do CRA foram avaliadas por meio de análise de componentes principais e de consistência interna, respectivamente. Correlações de Pearson entre escores do CRA e EBEP foram examinadas e utilizadas como indicadores de validade divergente e de construto. Resultados: As cinco escalas que compõem o CRA apresentaram bons níveis de unidimensionalidade e homogeneidade, porém as escalas de impacto nas finanças e impacto na saúde obtiveram alfas insuficientes $(<0,7)$. O escore total do CRA apresentou alfa elevado $(0,886)$. Correlações

\section{Palavras-chave}

Sobrecarga percebida, cuidador principal, câncer, bem-estar psicológico, hospitalização. entre o CRA e a EBEP produziram coeficientes teoricamente interpretáveis, com magnitudes variando entre nulas e moderadas. Conclusão: $O$ CRA apresentou bons indicadores de validade e fidedignidade. Algumas adaptações em relação ao conteúdo de determinados itens se mostram, todavia, necessárias, a fim de serem calibradas ao contexto de pessoas atendidas por serviços subsidiados pelo Sistema Único de Saúde.

\begin{abstract}
Objective: To adapt to Brazil a Portuguese version of the Caregiver Reaction Assessment (CRA) and generate preliminary indicators of validity and reliability for its application in caregivers of hospitalized cancer patients. Methods: Participated voluntarily 53 caregivers responding to a sociodemographic questionnaire, the CRA and the Psychological Well-Being Scale (PWS). Unidimensionality and homogeneity of the CRA scores were evaluated using principal component and internal consistency analysis, respectively. Pearson correlations between CRA and EBEP scores were examined and used as divergent and construct validity indicators. Results: The five scales that comprise the CRA showed good levels of unidimensionality and homogeneity, however the financial problems and loss of physical strength scales displayed insufficient alphas $(<0.7)$. The total score of CRA showed high alpha coef-
\end{abstract}

1 Universidade Federal de Pelotas (UFPel), Hospital-Escola, Programa de Residência Multiprofissional em Saúde - Atenção Oncológica. 2 UFPel, Departamento de Psicologia.

Recebido em $3 / 4 / 2014$ Aprovado em $1 / 9 / 2014$
Endereço para correspondência: Hudson W. de Carvalho Curso de Psicologia, Faculdade de Medicina, Universidade Federal de Pelotas

Av. Duque de Caxias, 250, Fragata 96030-001 - Pelotas, RS, Brasil E-mail: hdsncarvalho@gmail.com 


\section{Keywords}

Perceived overload, primary caregiver, cancer, psychological well-being, hospitalization. ficient (0.886). Correlations between the CRA and the PWS produced theoretically interpretable coefficients with magnitudes ranging between zero and moderate. Conclusion: The CRA showed good indicators of validity and reliability. Some adaptation on the content of certain items appears to be necessary to calibrate them to the context of people assisted by services subsidized by the Sistema Único de Saúde.

\section{INTRODUÇÃO}

O câncer é uma patologia definida com base no crescimento desordenado de células que invadem tecidos e órgãos e que podem se espalhar para outras regiões do corpo, causando a formação de tumores ou neoplasias malignas'. Sua manifestação está frequentemente associada à emergência de sofrimento psicológico clinicamente significativo² e à presença de psicopatologia ${ }^{3}$, particularmente aquelas relacionadas ao ajustamento e a sintomas depressivos e ansiosos.

Estimativas oficiais para os anos de 2012 e 2013 indicaram que a incidência de câncer na população brasileira foi de mais de 500 mil casos². Previsões internacionais antecipam uma incidência de 15 milhões de novos casos até o ano de 2020, e grande parte da incidência, morbidade e mortalidade deverão ocorrer em países em desenvolvimento como o Brasil ${ }^{3}$. A gravidade da condição e a crescente incidência dessa condição configuram claramente uma situação de saúde pública.

A fim de oferecer atenção integral ao paciente oncológico, é necessária a presença constante de uma pessoa - familiar ou não - que assuma o papel de cuidador principal. O cuidador é considerado a pessoa mais disponível para o paciente, oferecendo suporte emocional e cuidados no cotidiano ${ }^{4}$, o que o caracteriza como um coadjuvante na equipe de saúde e de essencial importância para o sucesso do tratamento.

O cotidiano do cuidador é influenciado pela constante necessidade de cuidados do paciente, o que pode afetar negativamente tanto a sua qualidade de vida quanto suas relações sociais, familiares e laborais 5 . A experiência do cuidado, quando vivenciada como árdua e negativa, pode estar associada à presença de psicopatologia ${ }^{3}$, que, por causa do foco no paciente e na gravidade de sua condição, tende a ser ignorada ou desvalorizada como uma situação de saúde que mereça atenção ${ }^{6}$. Nesse sentido, a utilização de instrumentos psicométricos breves e efetivos, voltados à avaliação do funcionamento emocional e social do cuidador principal, pode auxiliar a equipe de saúde a identificar problemas psicossociais relacionados às demandas do cuidar.

Um instrumento amplamente utilizado para avaliar os elementos positivos e negativos relacionados ao cuidado de pessoas portadoras de enfermidades graves é o Caregiver Reaction Assessment (CRA) ${ }^{7-10}$. Originalmente, o CRA foi desenvolvido por meio de um procedimento fatorial exploratório, no qual um conjunto de 40 itens foi desenhado para avaliar percepções subjetivas e crenças relativas ao cuidado de um paciente portador de doença crônica grave. Es- ses itens foram administrados a 377 cuidadores de pessoas idosos portadoras de alguma debilidade física, demência de Alzheimer ou câncer. A análise reduziu a 23 o número total de itens, que, por sua vez, se organizaram em cinco escalas que foram nomeadas conforme o conteúdo que avaliavam: autoestima do cuidador, falta de suporte familiar, impacto nas finanças, impacto no cotidiano e impacto na saúde. Os índices de consistência interna dos cinco escores apresentaram coeficientes alfa adequados $(>0,7)$, indicando alto grau de homogeneidade da medida ${ }^{7}$.

Ademais, o CRA se mostra uma boa opção para investigar a percepção subjetiva de sobrecarga do cuidador de pacientes graves, por razões de ordem tanto prática quanto conceitual. Primeiro, trata-se de um instrumento breve (23 itens), de aplicação simples e de uso livre, o que facilita sua utilização em diferentes serviços de saúde. Segundo, diferentes estudos atestam sua boa qualidade psicométrica9,10, o que indica que os escores produzidos com base em sua aplicação são razoavelmente confiáveis e teoricamente significativos. Por fim, o CRA avalia a percepção da sobrecarga do cuidador baseado em uma perspectiva multidimensional, que inclui aspectos tanto adaptativos (i.e., autoestima) quanto disruptivos (i.e., impacto na saúde) relacionados ao cuidar, permitindo abordar essa vivência de modo abrangente.

Desde a sua publicação, alguns estudos o têm utilizado a fim de avaliar a sobrecarga de cuidadores de pacientes com câncer em contextos ambulatoriais e de internação. Os dados, quando avaliados em conjunto, mostram que quanto maiores a gravidade da condição de saúde do paciente e a demanda por cuidado, maior a percepção de sobrecarga por parte do cuidador, sobretudo no que tange ao impacto no cotidiano e nas finanças ${ }^{11-15}$. Estudos que visam adaptar o CRA para outras culturas e fornecer dados sobre suas propriedades psicométricas em cuidadores de pacientes oncológicos têm mostrado que a estrutura pentafatorial do CRA é altamente replicável ${ }^{16-18}$, todavia há estudos que mostram indicadores insuficientes de fidedignidade por consistência interna para alguns de seus escores $(\geq 0,7)^{9,16}$.

Não há estudos no Brasil de adaptação e análise psicométrica do CRA, o que inviabiliza sua utilização em pesquisas ou em contextos de serviço de saúde. Assim sendo, o estudo ora apresentado teve como meta suplantar parcialmente a referida lacuna por meio da adaptação e da avaliação psicométrica inicial do CRA em uma amostra de cuidadores de pacientes oncológicos internados em um hospital público do sul do país. Procurou-se também explorar a relação en- 
tre percepção de sobrecarga e bem-estar psicológico, usando tais dados como uma medida de validade divergente ${ }^{19}$. Conjecturou-se que a versão adaptada ao Brasil apresentaria escalas unidimensionais e com bons indicadores de homogeneidade e que haveria uma relação inversamente proporcional entre percepção de sobrecarga e bem-estar psicológico. Por fim, o presente estudo também foi motivado pela possibilidade de equipar e qualificar a equipe responsável pela assistência ao paciente oncológico na instituição onde o estudo foi conduzido.

\section{MÉTODOS}

O presente estudo foi subdividido em dois momentos: o primeiro objetivou adaptar o CRA do português de Portugal para o do Brasil, e o segundo teve por meta administrar conjuntamente a versão adaptada do CRA e uma versão nacional validada da Escala de Bem-estar Psicológico (EBEP) ${ }^{20} \mathrm{em}$ uma amostra de cuidadores de pacientes oncológicos internados no Hospital-Escola da Universidade Federal de Pelotas (HE-UFPel). A escolha pela EBEP21 deveu-se a sua brevidade e ao fato de apresentar evidências recentes de validade e fidedignidade e de permitir complementar a avaliação de validade (divergente e de construto) do CRA ${ }^{19}$.

Em ambas as etapas, os participantes do estudo assinaram o Termo de Consentimento Livre e Esclarecido (TCLE) antes de responder aos questionários, elaborado com base nos requisitos da Resolução no 196/1996, do Conselho Nacional de Saúde do Brasil, e no código de ética da Associação Mundial de Medicina (Declaração de Helsinque). As participações foram voluntárias, sendo possível interrompê-las sem qualquer constrangimento ou necessidade de explicação. O projeto desta pesquisa foi aprovado pelo comitê de ética do HE-UFPel (Proc. Etic. no 386.007/UFPel).

\section{Etapa de adaptação do CRA}

A adaptação do CRA para o português brasileiro foi realizada com base em uma versão portuguesa do instrumento ${ }^{17}$. Visando à compreensão de estratos com níveis educacionais mais baixos, os itens foram adaptados de modo a privilegiar uma linguagem mais simples e direta. A tabela 1 traz as versões em inglês (original) e em português de Portugal e a adaptação para o português brasileiro do CRA.

Tabela 1. Itens das versões em inglês, português de Portugal e português do Brasil do CRA

\begin{tabular}{|c|c|}
\hline Versão em inglês & Versão em português/Portugal \\
\hline 1. I feel privileged to care for ____. & $\begin{array}{l}\text { 1. Sinto-me privilegiado(a) por cuidar do meu } \\
\text { familiar com DS. }\end{array}$ \\
\hline 2. Others have dumped caring for ____ onto me. & $\begin{array}{l}\text { 2. Os outros atiraram para cima de mim a responsabilidade } \\
\text { de cuidar do meu familiar com DS. }\end{array}$ \\
\hline $\begin{array}{l}\text { 3. My financial resources are adequate to pay for things that } \\
\text { are required for caregiving. }\end{array}$ & $\begin{array}{l}\text { 3. Os meus recursos financeiros são adequados para pagar o } \\
\text { que é necessário para os cuidados ao meu familiar com DS. }\end{array}$ \\
\hline 4. My activities are centered around care for __- & $\begin{array}{l}\text { 4. As minhas actividades são centradas ao redor no cuidar do } \\
\text { meu familiar com DS. }\end{array}$ \\
\hline 5. Since caring for____ it seems like I'm tired all the time. & $\begin{array}{l}\text { 5. Desde que cuido do meu familiar com DS, sinto-me } \\
\text { cansado o tempo todo. }\end{array}$ \\
\hline $\begin{array}{l}\text { 6. It is very difficult to get help from my family in taking care } \\
\text { of____-. }\end{array}$ & $\begin{array}{l}\text { 6. É bastante difícil arranjar ajuda da minha família para } \\
\text { cuidar do meu familiar com DS. }\end{array}$ \\
\hline 7. I resent having to care for & $\begin{array}{l}\text { 7. Eu sinto-me ressentido por ter de cuidar do meu familiar } \\
\text { com DS. }\end{array}$ \\
\hline 8. I have to stop in the middle of work. & $\begin{array}{l}\text { 8. Eu tenho que parar a meio do trabalho para cuidar do meu } \\
\text { familiar com DS. }\end{array}$ \\
\hline 9. I really want to care for & 9. Eu realmente quero cuidar do meu familiar com DS. \\
\hline 10. My health has gotten worse since I've been caring for & $\begin{array}{l}\text { 10. A minha saúde tem piorado desde que tenho cuidado do } \\
\text { meu familiar com DS. }\end{array}$ \\
\hline $\begin{array}{l}\text { 11. I visit family and friends less since I have been caring } \\
\text { for }\end{array}$ & $\begin{array}{l}\text { 11. Visito a família e amigos menos vezes desde que comecei } \\
\text { a cuidar do meu familiar com DS. }\end{array}$ \\
\hline 12. I will never be able to do enough caregiving to replay & $\begin{array}{l}\text { 12. Eu nunca me sentirei capaz de cuidar o suficiente do meu } \\
\text { familiar com DS. }\end{array}$ \\
\hline 13. My family works together at caring for ___-. & $\begin{array}{l}\text { 13. A minha família coopera nos cuidados do meu familiar } \\
\text { com DS. }\end{array}$ \\
\hline $\begin{array}{l}\text { 14. I have eliminated things from my schedule since caring } \\
\text { for }\end{array}$ & $\begin{array}{l}\text { 14. Tive que eliminar coisas do meu horário desde que cuido } \\
\text { do meu familiar com DS. }\end{array}$ \\
\hline 15. I have enough physical strength to care for & $\begin{array}{l}\text { 15. Tenho suficiente força física para cuidar do meu familiar } \\
\text { com DS. }\end{array}$ \\
\hline
\end{tabular}

Versão em português/Brasil

1. Sinto-me privilegiado por cuidar dele(a).

2. Os outros jogam em mim a responsabilidade de cuidar dele(a).

3. Tenho recursos financeiros suficientes para custear os cuidados que ele(a) precisa.

4. Minhas atividades giram em torno do cuidado com ele(a).

5. Desde que cuido dele(a) sinto-me cansado o tempo todo.

6. É muito difícil conseguir ajuda da minha família no cuidado com ele(a).

7. Sinto-me magoado por ter que cuidar dele(a).

8. Eu tenho que parar na metade do trabalho para cuidar dele(a).

9. Eu quero mesmo cuidar dele(a).

10. A minha saúde piorou desde que cuido dele(a)

11. Visito meus familiares e amigos menos desde que comecei a cuidar dele(a).

12. Eu nunca serei capaz de cuidar o suficiente dele(a).

13. A minha família ajuda no cuidado com ele(a).

14. Tive que tirar atividades da minha rotina desde que cuido dele(a).

15. Tenho força física suficiente para cuidar dele(a). 
Tabela 1. Itens das versões em inglês, português de Portugal e português do Brasil do CRA

\begin{tabular}{|c|c|c|}
\hline Versão em inglês & Versão em português/Portugal & Versão em português/Brasil \\
\hline 16. Since caring for____ I feel my family has abandoned me. & $\begin{array}{l}\text { 16. Desde que cuido do meu familiar com DS, sinto que a } \\
\text { minha família me abandonou. }\end{array}$ & $\begin{array}{l}\text { 16. Desde que cuido dele(a), sinto que minha família me } \\
\text { abandonou. }\end{array}$ \\
\hline $\begin{array}{l}\text { 17. The constant interruptions make it difficult to find time } \\
\text { for relaxation. }\end{array}$ & $\begin{array}{l}\text { 17. As constantes interrupções a que sou sujeito por cuidar } \\
\text { do meu familiar com DS tornam difícil encontrar tempo para } \\
\text { relaxar. }\end{array}$ & $\begin{array}{l}\text { 17. As constantes interrupções no meu dia a dia tornam } \\
\text { difícil encontrar tempo para descansar. }\end{array}$ \\
\hline 18. I am healthy enough to care for ____. & $\begin{array}{l}\text { 18. Sou suficientemente saudável para cuidar do meu } \\
\text { familiar com DS. }\end{array}$ & 18. Sou saudável o suficiente para cuidar dele(a). \\
\hline 19. Caring for ____ is important to me. & 19. Cuidar do meu familiar com DS é importante para mim. & 19. Cuidar dele(a) é importante para mim. \\
\hline 20. Caring for____ has put financial strain on my family. & $\begin{array}{l}\text { 20. Cuidar do meu familiar com DS tem criado dificuldades } \\
\text { financeiras na família. }\end{array}$ & $\begin{array}{l}\text { 20. Cuidar dele(a) tem deixado difícil a condição financeira } \\
\text { da minha família. }\end{array}$ \\
\hline $\begin{array}{l}\text { 21. My family (brothers, sisters and children) left me alone } \\
\text { to care for }\end{array}$ & $\begin{array}{l}\text { 21. Minha família (irmãos, irmãs e filhos) deixaram-me } \\
\text { sozinho(a) nos cuidados do meu familiar com DS. }\end{array}$ & $\begin{array}{l}\text { 21. Minha família (irmãos, irmãs, filhos) me deixaram } \\
\text { sozinho no cuidado com ele(a). }\end{array}$ \\
\hline 22. I enjoy caring for ___. & 22. Eu gosto muito de cuidar do meu familiar com DS. & 22. Eu gosto muito de cuidar dele(a). \\
\hline 23. It's difficult to pay for____'s health needs and services. & $\begin{array}{l}\text { 23. É difícil pagar as necessidades e serviços de saúde do meu } \\
\text { familiar com DS. }\end{array}$ & 23. É difícil pagar os serviços de saúde dele(a). \\
\hline
\end{tabular}

O método de inteligibilidade dos itens ${ }^{21}$ foi utilizado para avaliar o grau de entendimento dos participantes com relação ao conteúdo dos itens. Esse procedimento consiste em solicitar a um número restrito de participantes (entre cinco e dez) que respondam ao instrumento e, subsequentemente, retomem cada item, lendo-o novamente e relatando o seu entendimento. A resposta do participante é, então, avaliada pelo pesquisador quanto a sua acurácia. Quando o item se mostra ambivalente ou é compreendido de modo diferente do objetivo, é oferecida ao participante uma explicação do que se pretendia avaliar e the é requisitada uma sugestão de reformulação. Os itens que forem reformulados são novamente submetidos a um processo de análise de inteligibilidade.

Participaram dessa etapa, voluntariamente, cinco cuidadores de pacientes oncológicos que se encontravam acompanhando-os durante internação na Clínica Médica do HE-UFPel, durante o mês de agosto de 2013. Entre os participantes, três eram mulheres e dois homens, a média de idade foi de 46 anos e, em termos de estrato educacional, dois apresentavam ensino fundamental completo, um ensino médio completo e dois ensino superior incompleto. Todos os participantes mantinham vínculo próximo com o paciente internado (filho ou filha, esposa ou marido).

\section{Etapa de validação do CRA}

\section{Procedimentos}

Trata-se de um estudo de desenho transversal e de caráter psicométrico, baseado em uma amostra de conveniência. Inicialmente, a equipe de pesquisa entrou em contato com os pacientes oncológicos internados no HE-UFPel entre os meses de agosto e novembro de 2013 (período em que os dados foram coletados), a fim de apresentar a pesquisa e seus objetivos, identificar o cuidador principal, motivar a participação e obter TCLE.
Para participar do estudo, o cuidador deveria assinar o TCLE, ser alfabetizado e não apresentar algum transtorno neuropsiquiátrico ou físico que inviabilizasse sua participação. Com base na avaliação clínica realizada pela equipe de residentes da Psicologia do HE-UFPel e nos resultados do questionário sociodemográfico, não foram encontrados entre os potenciais participantes indivíduos analfabetos ou em condição de saúde que inviabilizasse a participação. A amostra resultante contou com 53 cuidadores, sendo oito homens $(15,1 \%)$ e 45 mulheres (84,9\%), com média etária de 45,96 anos (DP $=13,07)$ e predominantemente casados $(79,2 \%)$ ou solteiros (15,1\%). A maioria da amostra apresentava vínculo sanguíneo (49\%) ou marital com o paciente (34\%), e 43,4\% declararam ser os únicos cuidadores, não contando com auxílio de outrem, e que dedicava mais de 10 horas $(75,5 \%)$ ou entre 8 e 10 horas (24,5\%) por dia ao cuidado do paciente. Em termos de escolaridade formal, 58,5\% haviam concluído o ensino fundamental, 26,4\% terminaram o ensino médio e 15,1\% relataram estar cursando ou ter cursado o ensino superior. Apenas 11,3\% e 17\% apresentavam emprego formal ou estavam aposentados, respectivamente. A maioria era composta por indivíduos em situação de desemprego (37,7\%), trabalhos autônomos $(26,4 \%)$ ou informais $(5,7 \%)$ e que não dependiam financeiramente do paciente $(77,4 \%)$. A renda familiar se concentrou entre um e dois salários-mínimos $(73,6 \%)$ e três e cinco salários-mínimos (20,8\%); a minoria apresentava renda menor que um salário-mínimo (dois participantes, 3,8\%) ou entre cinco e dez salários-mínimos (um participante, 1,9\%). A tabela 2 traz o detalhamento da amostra organizada por sexo.

\section{Instrumentos}

Os dados demográficos foram coletados por meio de um questionário de autorrelato elaborado pela equipe de pesquisa. Seu objetivo foi o de avaliar variáveis como idade, sexo, estado civil, nível de escolaridade, profissão e situação 
profissional, renda familiar, diagnóstico físico e psiquiátrico, vínculo com o paciente, dependência financeira do cuidador em relação ao paciente, coabitação entre paciente e cuidador, o tempo dedicado ao cuidado do paciente e se o participante era único cuidador.

Tabela 2. Características sociodemográficas da amostra avaliada organizada por sexo

\begin{tabular}{|c|c|c|}
\hline & $\begin{array}{c}\text { Mulher } \\
\mathrm{n}(\%)\end{array}$ & $\begin{array}{c}\text { Homem } \\
\mathrm{n}(\%)\end{array}$ \\
\hline Média de idade (DP) & $47,2(13,4)$ & $39(8.2)$ \\
\hline \multicolumn{3}{|l|}{ Estado civil } \\
\hline Casado & $38(84,4)$ & $4(50)$ \\
\hline Solteiro & $4(8,9)$ & $4(50)$ \\
\hline Viúvo & $1(2,2)$ & \\
\hline Divorciado & $2(4,4)$ & \\
\hline \multicolumn{3}{|l|}{ Vínculo } \\
\hline Sanguíneo & $21(46,7)$ & $5(62,5)$ \\
\hline Marital & $17(37,8)$ & $1(12,5)$ \\
\hline Outro & $7(15,6)$ & $2(25)$ \\
\hline \multicolumn{3}{|l|}{ Único cuidador } \\
\hline $\operatorname{Sim}$ & $21(46,7)$ & $2(25)$ \\
\hline Não & $24(53,3)$ & $6(75)$ \\
\hline \multicolumn{3}{|c|}{ Horas/dia dedicadas ao cuidado } \\
\hline 8 horas & $5(11,1)$ & \\
\hline $8-10$ horas & $5(11,1)$ & $3(37,5)$ \\
\hline 10 horas ou mais & $35(77,8)$ & $5(62,5)$ \\
\hline \multicolumn{3}{|l|}{ Escolaridade } \\
\hline Ensino fundamental & $27(60)$ & $4(50)$ \\
\hline Ensino médio & $11(24,4)$ & $3(37,5)$ \\
\hline Ensino superior & $7(15,6)$ & $1(12,5)$ \\
\hline \multicolumn{3}{|l|}{ Situação profissional } \\
\hline Sem resposta & $1(2,2)$ & \\
\hline Empregado formal & $5(11,1)$ & $1(12,5)$ \\
\hline Empregado informal & $2(4,4)$ & $1(12,5)$ \\
\hline Desempregado & $15(33,3)$ & $5(62,5)$ \\
\hline Autônomo & $14(31,1)$ & \\
\hline Aposentado & $8(17,8)$ & $1(12,5)$ \\
\hline \multicolumn{3}{|l|}{ Dependência financeira } \\
\hline Sim & $11(24,4)$ & $1(12,5)$ \\
\hline Não & $34(75,6)$ & $7(87,5)$ \\
\hline \multicolumn{3}{|l|}{ Renda familiar } \\
\hline Sem resposta & $2(4,4)$ & \\
\hline 1 salário & $12(26,7)$ & $3(37,5)$ \\
\hline 2 salários & $22(48,9)$ & $2(25)$ \\
\hline 3-5 salários & $8(17,8)$ & $3(37,5)$ \\
\hline 5-10 salários & $1(2,2)$ & \\
\hline Total & $45(84,9)$ & $8(15)$ \\
\hline
\end{tabular}

O Caregiver Reaction Assessment7,18 é um instrumento que avalia aspectos específicos da situação de cuidar. Sua versão portuguesa é constituída por 23 itens que avaliam as reações do cuidador no cuidar de um paciente com uma doença física, em cinco dimensões: autoestima do cuidador, falta de suporte familiar, impacto nas finanças, impacto no cotidiano e impacto na saúde. Cada item é avaliado com base em uma escala do tipo Likert que varia entre concordo completamente até discordo completamente.

A EBEP é uma adaptação da versão de Ryff e Essex²2, sendo esta composta por 36 itens organizados em seis subescalas e um escore geral. Seus itens são mensurados em uma escala do tipo Likert de seis pontos, em que os extremos são discordo totalmente e concordo totalmente. O estudo brasileiro de adaptação e validação ${ }^{20}$ de suas escalas produziu bons indicadores de ajuste fatorial para um modelo de seis fatores correlacionados, além de coeficientes de consistência interna para cada escala se mostrarem adequados (entre 0,7 e 0,84). Desse modo, as dimensões da EBEP foram denominadas de modo semelhante a sua versão original, a saber: relações positivas com outros, autonomia, domínio sobre o ambiente, crescimento pessoal, propósito na vida, autoaceitação e bem-estar psicológico (escala geral).

\section{Procedimentos estatísticos}

A unidimensionalidade e a homogeneidade dos escores do CRA foram avaliadas por meio de análise de componentes principais, usando-se o critério de autovalor acima de 1,0 para retenção de componentes, e de cálculo do coeficiente alfa de Cronbach, respectivamente ${ }^{23}$. Foram consideradas unidimensionais escalas que apresentavam apenas um componente principal com autovalor acima de 1,0 e homogêneas as escalas com coeficientes alfa acima de 0,7. A pertinência do uso de análise de componentes principais foi avaliada pelo valor da medida de Kaiser-Meyer-Okin (KMO) de adequação da amostra $(K M O>0,6)$ e do teste de esfericidade de Bartlett $(p \leq, 05)^{23}$.

Por causa do restrito número amostral e com o intuito de aumentar o poder estatístico, as análises de componentes principais foram realizadas de modo independente para cada escala/escore do CRA. Esse procedimento se mostra pertinente, uma vez que o objetivo aqui é o de apenas avaliar a unidimensionalidade de cada escala e, desse modo, justificar a viabilidade dos escores das escalas da EBEP.

A análise de validade divergente foi realizada mediante correlações bivariadas de Pearson entre os escores do CRA e da EBEP, sendo consideradas significantes quando apresentassem um valor de $p$ menor que 0,05. Coeficientes teoricamente interpretáveis (i.e. relação inversa entre bem-estar e indicadores negativos de sobrecarga), com tamanho de efeito variando entre suaves $(r \approx 0,2)$ e moderados $(r \approx 0,4)$, foram considerados como bons indicadores de validade divergente ${ }^{19}$. 


\section{RESULTADOS}

\section{Unidimensionalidade e homogeneidade das escalas}

Os valores encontrados para a medida de KMO de adequação da amostra e para o teste de esfericidade de Bartlett foram, para todas as escalas do CRA, superiores a 0,7 e significantes ( $p \leq, 05)$, respectivamente. Esses resultados atestaram a pertinência do uso de análises de componentes.

Os resultados referentes às análises de componentes principais apontaram que todas as escalas que compõem o CRA apresentam indicadores adequados de unidimensionalidade com base no critério de autovalor acima de 1,0. As escalas de autoestima do cuidador (alfa $=, 905$ ), falta de suporte familiar (alfa $=, 794)$ e impacto no cotidiano (alfa $=, 786)$ apresentaram coeficientes de consistência interna adequados, enquanto as escalas de impacto nas finanças (alfa $=, 561)$ e impacto na saúde (alfa = ,686) apresentaram alfas insuficientes. O escore total do CRA apresentou um alfa elevado (alfa = ,886). A tabela 3 apresenta o detalhamento dos resultados da análise de unidimensionalidade e homogeneidade.

\section{Análises de validade divergente}

Análises de correlação entre os escores produzidos com base na administração do CRA e EBEP produziram coeficientes que variam entre não significantes e moderados, oferecendo indícios de validade divergente para o CRA. Autoestima do cuidador mostrou correlações leves com autonomia $(r=.32)$, propósito na vida $(r=.34)$ e bem-estar psicológico $(r=.30)$. Falta de suporte familiar apresentou coeficientes moderados e negativos com relações positivas com os outros $(r=-.43)$, propósito na vida $(r=-.45)$ e autoaceitação

Tabela 3. Análise de componentes principais e de consistência interna do Caregiver Reaction Assessment (CRA)

\begin{tabular}{|c|c|c|c|c|c|c|}
\hline \multirow{2}{*}{ Item } & \multirow{2}{*}{ Conteúdo } & \multicolumn{5}{|c|}{ Componentes principais } \\
\hline & & Autoestima & Família & Finanças & Cotidiano & Saúde \\
\hline 09 & Querer cuidar & 0,95 & & & & \\
\hline 19 & Cuidar é importante & 0,92 & & & & \\
\hline 22 & Gosto de cuidar & 0,95 & & & & \\
\hline 01 & Privilegiado por cuidar & 0,84 & & & & \\
\hline 19 & Magoado por cuidar & 0,70 & & & & \\
\hline 22 & Cuidar me faz bem & 0,60 & & & & \\
\hline 12 & Ajuda da família & & 0,85 & & & \\
\hline 02 & Obrigação no cuidado & & 0,84 & & & \\
\hline 06 & Difícil conseguir ajuda & & 0,81 & & & \\
\hline 21 & Sozinho no cuidado & & 0,76 & & & \\
\hline 15 & Abandono da família & & 0,41 & & & \\
\hline 23 & Difícil pagar os serviços & & & 0,89 & & \\
\hline 20 & $\begin{array}{l}\text { Condição financeira } \\
\text { precária }\end{array}$ & & & 0,80 & & \\
\hline 03 & $\begin{array}{l}\text { Recursos financeiros } \\
\text { disponíveis }\end{array}$ & & & 0,45 & & \\
\hline 04 & Centro das atividades & & & & 0,78 & \\
\hline 17 & Difícil descansar & & & & 0,76 & \\
\hline 08 & $\begin{array}{l}\text { Interrupção das } \\
\text { atividades }\end{array}$ & & & & 0,72 & \\
\hline 13 & $\begin{array}{l}\text { Menor número de } \\
\text { atividades }\end{array}$ & & & & 0,72 & \\
\hline 11 & Menos visitas familiares & & & & 0,69 & \\
\hline 05 & Cansaço & & & & & 0,83 \\
\hline 10 & Pior condição de saúde & & & & & 0,72 \\
\hline 18 & Saudável suficiente & & & & & 0,70 \\
\hline 14 & Força física & & & & & 0,61 \\
\hline Autovalor & & 4,15 & 2,84 & 1,65 & 2,7 & 2,07 \\
\hline Variância (\%) & & 69,14 & 56,75 & 54,98 & 53,94 & 51,92 \\
\hline Coeficiente alfa & & 0,89 & 0,80 & 0,57 & 0,78 & 0,68 \\
\hline
\end{tabular}


( $r=-.40)$ e leves com domínio sobre o ambiente $(r=-.34)$ e bem-estar psicológico $(r=-.39)$. Impacto nas finanças apresentou coeficientes leves e negativos com domínio sobre o ambiente ( $r=-.32)$ e propósito na vida $(r=-.28)$. Impacto no cotidiano apresentou associação somente com a escala de domínio sobre o ambiente ( $r=-.27)$. Impacto na saúde apresentou associações moderadas e negativas com as escalas domínio sobre o ambiente $(r=-.45)$, propósito na vida $(r=-.52)$, autoaceitação $(r=-.45)$ e bem-estar psicológico ( $r=$ $-.44)$ e leve com crescimento pessoal $(r=-.30)$. A sobrecarga de modo geral apresentou coeficientes moderados e negativos com domínio sobre o ambiente $(r=-.45)$, propósito na vida ( $r=-.49)$, autoaceitação $(r=-.41)$ e bem-estar psicológico ( $r=-.41)$, e correlação leve com relação positiva com os outros $(r=-28)$. A tabela 4 traz a matriz de correlação entre as variáveis avaliadas.

Tabela 4. Matriz de correlações bivariadas entre escores do Caregiver Reaction Assessment (CRA) e da Escala de Bem-Estar Psicológico (EBEP)

\begin{tabular}{|c|c|c|c|c|c|c|c|}
\hline & RP & AUT & DA & CP & PV & AA & BEP \\
\hline$A C$ & .10 & $.32^{*}$ & .25 & .10 & $.34^{*}$ & .12 & $.30^{*}$ \\
\hline Familiar & $-.43^{*}$ & -.17 & $-.34^{*}$ & -.10 & $-.45 *$ & $-.40 *$ & $-.39 *$ \\
\hline Finanças & -.12 & -.12 & $-.32^{*}$ & -.12 & $-.28^{*}$ & -.25 & -.17 \\
\hline Cotidiano & -.09 & -.02 & $-.27^{*}$ & -.11 & -.19 & -.22 & -.18 \\
\hline Saúde & -.22 & -.14 & $-.45^{*}$ & $-.30^{*}$ & $-.52^{*}$ & $-.45^{*}$ & $-.44^{*}$ \\
\hline Sobrecarga & $-.28^{*}$ & -.13 & $-.45^{*}$ & -.20 & $-.49 *$ & $-.41^{*}$ & $-.41^{*}$ \\
\hline
\end{tabular}

RP: relação positiva com outros; AUT: autonomia; DA: domínio sobre o ambiente; (P: crescimento pessoal; PV: propósito na vida; AA: autoaceitação; BEP: bem-estar psicológico. Coeficientes significantes $(p \leq, 05)$ estão destacados pelo* e em negrito.

\section{DISCUSSÃO}

O presente estudo teve como meta adaptar e avaliar algumas propriedades psicométricas do CRA em uma amostra de cuidadores de pacientes oncológicos internados em um hospital-escola do sul do país. Pretendeu-se, ainda, entender a relação entre os aspectos positivos e negativos da percepção de sobrecarga e dimensões de bem-estar psicológico na amostra avaliada.

Os resultados referentes à análise de componentes principais indicaram que os itens designados para avaliar cada uma das escalas que conformam o CRA se mostraram unidimensionais. Além disso, as escalas de autoestima do cuidador, falta de suporte familiar e impacto no cotidiano apresentaram coeficientes de consistência interna/homogeneidade adequados $(\alpha>0,7)$, enquanto impacto nas finanças $(\alpha=0,561)$ e impacto na saúde $(\alpha=0,686)$ obtiveram alfas insuficiente e limítrofe, respectivamente. Tais achados são comparáveis aos de pesquisas internacionais ${ }^{9,15-17}$, o que mostra que há necessidade de adaptar itens do ponto de vista cultural - particularmente no que tange à escala de impacto na finanças, mesmo que isso represente alterar o significado do item em questão.
Pontua-se, adicionalmente, que o baixo coeficiente $\alpha$ da escala impacto nas finanças pode se dever a dois aspectos, sendo o primeiro relacionado à amostragem e o segundo, à baixa sensibilidade do instrumento em diferenciar pessoas com baixo padrão socioeconômico. Como 77,4\% dos participantes apresentavam baixa renda familiar (até dois salários-mínimos) e como o serviço de assistência prestado é subsidiado pelo Sistema Único de Saúde, espera-se baixa variabilidade de respostas. A título de exemplo, o item "Tenho recursos financeiros suficientes para custear os cuidados que ele(a) precisa" apresentou baixa variabilidade, e sua exclusão elevaria o coeficiente alfa para 0,72. Sugere-se, desse modo, a reformulação desse item e a possível inclusão de outros que representem com mais verossimilhança as características da população usuária do Sistema Único de Saúde.

Já a escala impacto na saúde pode ter obtido um alfa limítrofe em virtude de uma restrição do conteúdo avaliado pelos itens, o que a variabilidade de respostas. Os itens dessa escala focam na percepção de fadiga, disponibilidade física para o cuidado e saúde física de modo genérico, deixando de especificar manifestações de mal-estar e sobrecarga como insônia, ansiedade, depressão, dores nas articulações, entre outras. Sugere-se a inclusão de itens com maior grau de especificidade e amplitude, incluindo sintomas físicos e psicológicos referentes ao contexto de cuidado.

De fato, a avaliação qualitativa das respostas dadas aos itens do CRA mostra tendência dos participantes em concordar completamente com a afirmação que conforma o item ou discordar completamente dela. Isso pode estar relacionado ao baixo padrão educacional dos participantes, uma vez que a natureza verbal do teste demanda maior grau de discriminação semântica por parte do respondente. Todavia, a fim de aprimorar as qualidades psicométricas do CRA no que tange à avaliação de usuários do Sistema Único de Saúde, sugere-se uma avaliação qualitativa do conteúdo dos itens, com o intuito de ajustá-los à realidade de pessoas com menor grau de escolaridade e de baixa renda.

As associações entre os escores do CRA e da EBEP apresentaram coeficientes de correlações com magnitude e direção conceitualmente pertinentes: quanto maior a autoestima, maior o senso de autonomia, propósito na vida e bem-estar psicológico; quanto maior a falta de suporte familiar, menor a percepção de relações positivas com os outros, propósito na vida, domínio sobre o ambiente, autoaceitação e bem-estar psicológico; quanto maior o impacto nas finanças, menor a noção de domínio sobre o ambiente e propósito na vida; quanto maior o impacto no cotidiano, menor o senso de domínio sobre o ambiente; quanto maior o impacto na saúde, menor a percepção de domínio sobre o ambiente, propósito na vida, autoaceitação, bem-estar psicológico e crescimento pessoal (Tabela 4). As facetas do bem-estar psicológico mais associadas à percepção de sobrecarga foram propósito na vida e domínio sobre o ambiente, e a faceta 
da sobrecarga mais associada a baixo bem-estar psicológico foi o impacto na saúde. Esses resultados permitem conjecturar que a percepção de sobrecarga - particularmente a relacionada à saúde - está associada a um esvaziamento do indivíduo enquanto agente da própria vida, manifestado principalmente pela falta de metas pessoais, sentido de vida, habilidade em planejar e fazer uso efetivo do ambiente e construir um ambiente que seja favorável a si próprio. A fim de lidar com a referida situação, pode ser importante que a equipe de saúde mantenha um vínculo com serviços de atendimento psicoterapêutico.

Outra interpretação desses resultados se refere à noção de validade divergente. As associações entre CRA e EBEP apresentaram uma magnitude que variou entre nula e moderada, evidenciando que os instrumentos avaliam construtos diferentes.

Algumas limitações deste estudo merecem ser ressaltadas. A primeira se refere às restrições relacionadas à amostra. Em virtude do limitado número de participantes, não foi possível avaliar a estrutura do instrumento considerando todos os itens simultaneamente ou por meio da utilização de técnicas estatísticas mais avançadas, como análise fatorial confirmatória e teoria de resposta ao item. Outro aspecto importante é que o caráter de conveniência da amostra não permite que os achados sejam amplamente generalizados, atribuindo-se à presente pesquisa o status de estudo preliminar. Por fim, o desenho transversal do estudo permite apenas fazer inferências de associação, sendo metodologicamente impossível estabelecer uma relação de causa-efeito entre percepção de sobrecarga e bem-estar psicológico.

Entre suas virtudes, ressalta-se a repercussão prática para o serviço de saúde onde foi realizado o estudo e seu valor heurístico. As informações aqui geradas foram utilizadas para elaborar o protocolo básico de atendimento de psicologia do programa de atenção integral ao paciente oncológico do hospital-escola da UFPel. A adaptação do CRA potencializa a efetivação de outros estudos e de aplicações clínicas para o contexto hospitalar.

Por fim, os dados ora apresentados, quando concatenados com os de outros estudos ${ }^{5,24}$, enfatizam a importância de focar no bem-estar do cuidador principal do paciente com câncer e intervir preventivamente. Mais especificamente, intervenções psicossociais (por exemplo, psicoterapia individual e de grupo ou acompanhamento terapêutico) podem ser fundamentais ao fomentarem estratégias de enfrentamento que estimulem atitudes mais proativas e resolutivas diante dos impasses relacionados ao papel de cuidador principal.

\section{CONCLUSÃO}

O CRA apresenta boas propriedades psicométricas quanto à unidimensionalidade e à consistência interna da maioria de seus escores. As escalas de impacto nas finanças e impacto na saúde necessitam de algumas modificações, a fim de aprimorar seu grau de homogeneidade. A percepção de sobrecarga e o bem-estar psicológico se mostram associados, mas sobrecarga de saúde parece ser a faceta mais negativamente associada ao bem-estar psicológico. Apesar de preliminares, os resultados indicam que a versão adaptada para o Brasil do CRA pode ser útil na avaliação da percepção de sobrecarga do cuidador principal de pacientes oncológicos.

\section{CONTRIBUIÇÕES INDIVIDUAIS}

\section{Suzane Pereira Busatta e Renata Alexandre Ferreira -}

Coletaram e tabularam os dados e participaram da redação do manuscrito e da discussão dos resultados.

Luiz Gustavo Klumb Kiesow - Realizou o levantamento bibliográfico e participou da redação do manuscrito e da discussão dos resultados.

Hudson W. de Carvalho - Elaborou o projeto, analisou os dados e participou da redação do manuscrito, da discussão dos resultados e da revisão da versão final do artigo.

\section{CONFLITOS DE INTERESSE}

Os autores relatam não ter conflitos de interesse. Suzane Pereira Busatta e Renata Alexandre Ferreira foram bolsistas de residência da Coordenação de Aperfeiçoamento de Pessoal de Nível Superior (Capes) durante a elaboração do presente trabalho e Luiz Gustavo Klumb Kiesow é bolsista de iniciação científica da Fundação de Amparo à Pesquisa do Estado do Rio Grande do Sul (Fapergs).

\section{AGRADECIMENTOS}

Agradecemos à Capes e à Fapergs pelo auxílio aos pesquisadores deste trabalho.

\section{REFERÊNCIAS}

1. Instituto Nacional de Câncer José Alencar Gomes da Silva (Inca), Ministério da Saúde. ABC do câncer - Abordagens básicas para o controle do câncer. $2^{\text {a }}$ ed. revista e atualizada [online]. Rio de Janeiro, RJ: Inca; 2012.

2. Instituto Nacional de Câncer José Alencar Gomes da Silva (Inca), Ministério da Saúde. Estimativa 2012: incidência de câncer no Brasil [online]. Rio de Janeiro, RJ: INCA; 2011.

3. Kanavos P. The rising burden of cancer in the developing world. Ann 0ncol. 2006;17(Supp 8):15-23.

4. Sales $(A$, Matos PCB, Mendonça DPR, Marcon SS. Cuidar de um familiar com câncer: 0 impacto no cotidiano de vida do cuidador. Rev Eletr Enferm. 2010;12(4):616-21.

5. Sena EL, Carvalho PAL, Reis HFT, Rocha MB. Percepção de familiares sobre o cuidado à pessoa com câncer em estágio avançado. Texto Contexto Enferm. 2011;20(4):774-81. 
6. Sorato DB, Peres SV, Mitsuyuki MC, Drude FS. Cuidar e ser cuidado pelo grupo de apoio protege. Psicol Estud. 2010;15(4):751-9.

7. Given CW, Given B, Stommel M, Collins C, King S, Franklin S. The Caregiver Reaction Assessment (CRA) caregivers to persons with chronic physical and mental impairments. Res Nurs Health. 1992;15(4):271-83.

8. Rezende VL, Derchain S, Botega NJ, Sarian LO, Vial DL. Avaliação psicológica dos cuidadores de mulheres com câncer pelo General Comfort Questionnaire. Paideia. 2010;20(46):229-37.

9. Nijboer C, Triemstra M, Tempelaar R, Sanderman R, Van Den Bos G. Measuring both negative and positive reactions to giving care to cancer patients: Psychometric qualities of the Caregiver Reaction Assessment (CRA). Soc Sci Med. 1999;48(9):1259-69.

10. Grov EK, Dahl AA, Moum T, Fossa SD. Anxiety, depression, and quality of life in caregivers of patients with cancer in late palliative phase. Ann Oncol. 2005;16(7):1185-91.

11. Given CW, Stommel M, Given B, Osuch J, Kurtz ME, Kurtz JC. The influence of cancer patient symptoms, functional states on patient depression and family caregiver reaction and depression. Health Psychol. 1993;12(4):277-85.

12. Kurtz ME, Given B, Kurtz JC, Given CW. The interaction of age, symptoms, and survival status on physical and mental health of patients with cancer and their families. Cancer. 1994;74(7 Suppl):2071-8.

13. Kurtz ME, Kurtz JC, Given CW, Given B. Relationship of caregiver reactions and depression to cancer patients' symptoms, functional states and depression: a longitudinal view. Soc Sci Med. 1995;40(6):837-44.

14. Milbury K, Badr H, Fossella F, Pisters KM, Carmack CL. Longitudinal associations between caregiver burden and patient and spouse distress in couples coping with lung cancer. Support Care Cancer. 2013;21(9):2371-9.
15. Sautter JM, Tulsky JA, Johnson KS, Olsen MK, Burton-Chase AM, Lindquist JH, et al. Caregiver experience during advanced chronic illness and last year of life. J Am Geriatr Soc. 2014;62(6):1082-90.

16. Grov EK, Fossa SD, Tønnessen T, Dahl A. The caregiver reaction assessment: psychometrics, and temporal stability in primary caregivers of Norwegian cancer patients in late palliative phase. Psychooncology. 2006;15(6):517-27.

17. Yang HK, Shin DW, Kim SY, Cho J, Chun SH, Son KY, et al. Validity and reliability of the Korean version of the caregiver reaction assessment scale in family caregivers of cancer patients. Psychooncology. 2013;22(12):2864-8.

18. Pereira MG, Soares AJ. Sobrecarga em cuidadores informais de dependentes de substâncias: adaptação do Caregiver Reaction Assessment (CRA). Psic Saúde Doenças. 2011;12(2):304-28

19. Urbina S. Fundamentos da testagem psicológica. Porto Alegre, RS: Artmed; 2007.

20. Machado WL, Bandeira DR, Pawlowski J. Validação da Psychological Well-being Scale em uma amostra de estudantes universitários. Aval Psicol. 2013;12(2):263-72.

21. De Carvalho HW, Pinheiro AMV, Patrick CJ, Krueger RF, Markon KE. Tradução, adaptação cultural e análise de consistência interna do Inventário de Externalização. Aval Psicol. 2007;6(2):217-27.

22. Ryff CD, Essex MJ. The interpretation of life experience and well-being: the sample case of relocation. Psychol Aging. 1992; 7(4):507-17.

23. Hair JF, Anderson RE, Tathan RL, Black WC. Análise multivariada de dados. Porto Alegre, RS: Bookman; 2005.

24. Rabow MW, Hauser JM, Adams J. Supporting family caregivers at the end of life: "They don't know what they don't know". JAMA. 2004;291(4):483-91. 